\title{
FPGA IMPLEMENTATION OF COMB FILTER FOR AUDIBILITY ENHANCEMENT OF HEARING IMPAIRED
}

\author{
J.J. Chopade ${ }^{1}$, N.P. Futane ${ }^{2}$ and Viral N. Shah ${ }^{3}$ \\ ${ }^{I}$ Department of Electronics and Telecommunication Engineering, Matoshri College of Engineering and Research Center, India \\ ${ }^{2}$ Department of Electronics and Telecommunication Engineering, Government College of Engineering and Research, India \\ ${ }^{3}$ Veer Technologies, India
}

\begin{abstract}
In this paper, FPGA implementation of comb filter required for hearing aids useful for hearing impaired people suffering from sensorineural hearing loss has been proposed. The difficulties in algorithm implementation on FPGA based hardware is the complexity involved during design and it implementation. Here, a new approach has been used to implement comb filter on FPGA platform. This work describes the hardware miniaturization of the hearing aid system.
\end{abstract}

Keywords:

Sensorineural Hearing Loss, Comb Filter, FPGA

\section{INTRODUCTION}

Human ear consists of outer, middle and inner ear. According to the damage in the ear, hearing impaired are classified as conductive and sensorineural hearing impairment. Conventional hearing aid improves the problems of elevated thresholds, found in conductive hearing impairment, and does not provide improvement in qualitative speech presentation for sensorineural hearing-impaired people. Sensorineural hearing impairment takes place due to damage to the transduction mechanism of the inner ear. The problem of sensorineural hearing loss can be solved by development of digital hearing aids instead of going for surgical treatments [1]. Advancements in microelectronics and digital signal processing algorithms have given rise to superior digital hearing aids. System applications are miniaturized by improvement in field programmable gate array (FPGA) technology which allows development of increase in sophisticated features, reducing the size and lowering the power consumption of the devices.

The audibility enhancement of sensorineural hearing-impaired people is improved using comb filters [4]. Perceptually balanced comb filter with critical bandwidth based helps in reducing the spectral masking $[4,6]$. The spectral splitting approach is obtained using pair of liner phase FIR comb filter with complimentary magnitude response for binaural dichotic presentation [4]-[6], which improves the perception of moderate bilateral loss hearing impaired people.

Earlier literature investigation shows that the DSP processors are being used to implement the FIR filter banks. Few researchers have attempted to implement filter algorithm on FPGA. Lunar and Hellgren [5] implemented an 8-channel digital filter bank on DSP processor. Filters implemented on DSP processor were complementary interpolated linear phase FIR filters of $700 \mathrm{~Hz}$ of constant bandwidth. In the literature [6], the increase of frequency bands to eighteen has been proposed. The research work has been carried out in real time using two TI/TMS320C50 DSP processors. Ghamry [10] has developed algorithm using stationary wavelet packets with noise reduction for hearing impaired. The processing core is implemented on Xilinx FPGA XC2VP30 containing LM4550 (audio codec). Derouiche [12] filter using wavelet techniques was implemented on FPGA (Virtex5) for cochlear implant people. Kambalimath et al. [13] conveyed that for applications concerning highly parallel computations, FPGAs are more suitable than Digital signal processing chips.

The objective of this paper is to miniaturize the filter implementation system. In this paper, an attempt to implement the comb filter using FPGA has been proposed. For the scheme of spectral splitting, a pair of complementary comb filters, from the filter sets has been used. These pair of comb filters have nine pass bands consistent with auditory critical bandwidths filters [6]. The comb filter is designed using direct form-1 structure with basic principle of convolution and with two inputs basic adder, adder tree is used to add the result of all multiplications involved. The HDL coder is used to convert MATLAB code into synthesizable VHDL code for FPGA implementation. The HDL code is optimized for hardware implementation and verified. The RTL schematic and simulated results of generated VHDL code is been presented and discussed.

The paper is organized with introduction in section 1 , section 2 discusses the methodology used for implementation and results are presented in section 3. Finally, conclusion is presented in section 4 .

\section{METHODOLOGY}

The methodology uses spectral splitting with comb filters based on auditory critical bandwidths and the responses obtained are optimized for low perceived spectral distortion.

For binaural splitting of speech signal, filters were designed using linear phase FIR filters. Frequency sampling technique was used to design these filters [2], [3]. In the frequency sampling technique, the desired frequency response $H\left(e^{j \omega}\right)$ is sampled at a set of uniformly spaced frequencies,

$$
\omega=2 \pi k / N
$$

where,

$k=0,1,2, \ldots,(N-1) / 2$, for odd values of $N$ and $k=0,1,2, \ldots$, (N/2)-1, for even values of $N$.

The filter coefficients of these set of samples are determined using Inverse Discrete Fourier transform (IDFT) as,

$$
h(n)=\frac{1}{N} \sum_{k=0}^{N-1} H(k) e^{\frac{j 2 \pi n k}{N}} \text { for } n=0,1,2, \ldots, N-1
$$


The frequency response $H\left(e^{j \omega}\right)$, calculated using $N$-point Finite Impulse Response (FIR) $h(n)$, coincides with $H_{d}\left(e^{j \omega}\right)$, at $\omega$ $=2 \pi k / N$ is formulated as,

$$
H\left(e^{j \omega}\right)=\sum_{n=0}^{N-1} h(n) e^{-j \omega n}
$$

The $L$ uniformly spaced samples of the frequency response $H\left(e^{j \omega}\right)$ are given as:

$$
H\left(e^{\frac{j 2 \pi k}{L}}\right)=\sum_{n=0}^{N-1} h(n) e^{-\frac{j 2 \pi k n}{L}} \text { for } k=0,1,2, \ldots, L-1
$$

A MATLAB program was developed to generate 512-filter coefficients of comb filter. In initial stage, 512 coefficient comb filters were designed. The Fig. 1 shows the magnitude response of the designed filters having nine pass-bands corresponding to auditory critical bandwidths [7]. The auditory critical bandwidths having center frequency $\left(F_{\text {centre }}\right)$ and pass-band frequency $\left(F_{\text {pass }}\right)$ upto $5 \mathrm{kHz}$ are shown in Table. 1 .

Table.1. Eighteen bands as per Critical Bandwidth

\begin{tabular}{|c|c|c|}
\hline Band & $\boldsymbol{F}_{\text {center }}(\mathbf{k H z})$ & $\boldsymbol{F}_{\text {pass }}(\mathbf{k H z})$ \\
\hline 1 & 0.13 & $0.01-0.20$ \\
\hline 2 & 0.25 & $0.20-0.30$ \\
\hline 3 & 0.35 & $0.30-0.40$ \\
\hline 4 & 0.45 & $0.40-0.51$ \\
\hline 5 & 0.57 & $0.51-0.63$ \\
\hline 6 & 0.70 & $0.63-0.77$ \\
\hline 7 & 0.84 & $0.77-0.92$ \\
\hline 8 & 1.00 & $0.92-1.08$ \\
\hline 9 & 1.17 & $1.08-1.27$ \\
\hline 10 & 1.37 & $1.27-1.48$ \\
\hline 11 & 1.60 & $1.48-1.72$ \\
\hline 12 & 1.86 & $1.72-2.00$ \\
\hline 13 & 2.16 & $2.00-2.32$ \\
\hline 14 & 2.51 & $2.32-2.70$ \\
\hline 15 & 2.92 & $2.70-3.15$ \\
\hline 16 & 3.42 & $3.15-3.70$ \\
\hline 17 & 4.05 & $3.70-4.40$ \\
\hline 18 & 4.70 & $4.40-5.00$ \\
\hline
\end{tabular}

However, the filter designed using MATLAB HDL coder was not properly synthesized into FPGA. Therefore, a new approach for designing the comb filter for synthesizable FPGA implementation is been proposed here. The direct form-I filter approach, using the generated coefficients, is been used for designing the odd and even filter bank which uses the basic steps of convolution, as shown in Fig.2. Since the basic adder has only two inputs, the adder tree is used to add results of all multiplication. This approach makes the MATLAB code synthesizable into FPGA.

The transfer function used for implementation is given as, if filter input and output are $X(z)$ and $Y(z)$ are respectively, then $H(z)$ is given as:

$$
H(z)=\frac{Y(z)}{X(z)}=\sum_{i=0}^{N} a_{i} z^{-i}
$$

where, $a_{i}$ is a filter coefficient hence $N$ is order of filter and $N+1$ is length of filter. The difference equation for odd filter bank is given by Eq.(2).

$$
\text { Out }_{h}=h(0) u(0)+h(1) u(n-1)+\ldots .+h(512) u(n-512)
$$

where, the length of filter is 513 and order of filter is 512 and $h(0)$, $h(1), \ldots, h(512)$ are odd filter bank coefficient. Similarly, the difference equation for even filter bank is given by Eq.(7).

$$
O u t_{g}=g(0) u(0)+g(1) u(n-1)+\ldots .+g(512) u(n-512)
$$
where, $g(0), g(1), \ldots, g(512)$ are even filter bank coefficients.

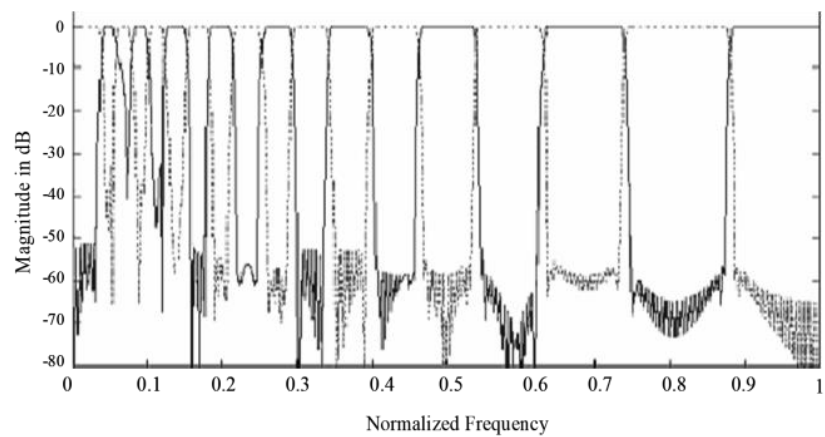

Fig.1. Magnitude response of comb filter

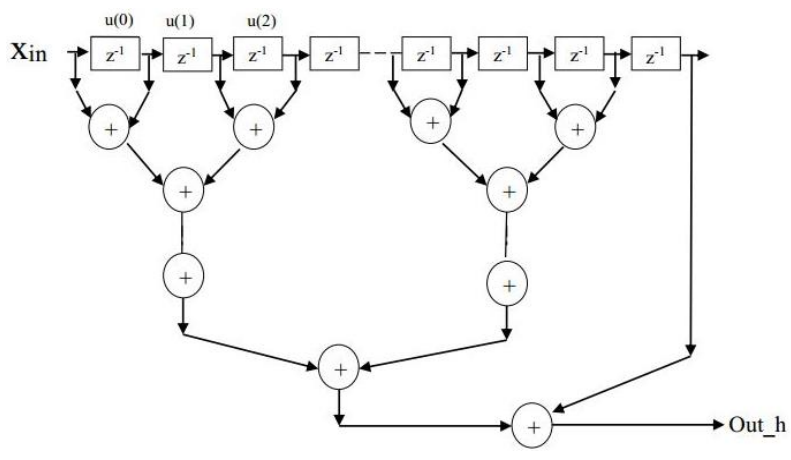

Fig.2. Filter Structure

\section{IMPLEMENTATION OF FILTER AND RESULTS}

The eighteen bands obtained from auditory critical bandwidths using pair of complementary comb filters from the filter sets were used to implement the algorithm. An input speech signal in vowel consonant vowel (VCV) context is fed to the system. The filter bank splits the input speech signal into even and odd bands. The magnitude response for critical band filter using MATLAB code for two input speech signals are shown in Fig. 3 and Fig.4 respectively.

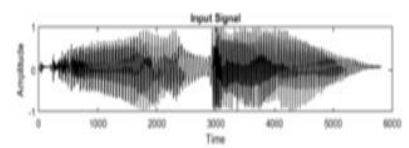

(a)

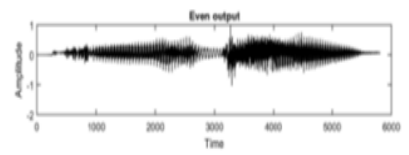

(b) 


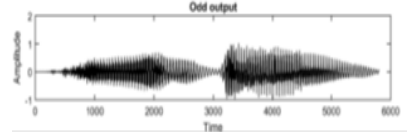

(c)

Fig.3. (a) Unprocessed signal b) Processed ouput of even bands for right ear c) Processed ouput of odd bands for left ear for input 'ada.wav' speech signal

Once the MATLAB simulated results are verified, the HDL coder is used to convert the MATLAB code into VHDL code. The generated VHDL code is synthesized and the RTL schematic is generated using XILINX 14.2 for verification. The generated RTL schematic for comb filter is shown in Fig.5 and detailed RTL schematic is shown in Fig.6. The generated RTL schematic shows that the implemented filter structure in FPGA have 14-bit input called $x_{i n}$. The output signal obtained for even and odd bands are namely $g_{\text {out }}$ and $h_{\text {out }}$ respectively, both of 14 bits. The comb filter has been implemented on Xilinx Spartan-6 FPGA. The Fig.7 shows the simulated test bench waveform using ModelSim for the implemented comb filter. The implementation of this filter utilized $7 \%$ of total slice registers, $88 \%$ of slice LUT's, $5 \%$ of LUT-FF pairs and $11 \%$ of bonded IOB's, indicating a scope to implement additional processing blocks required for hearing aid.

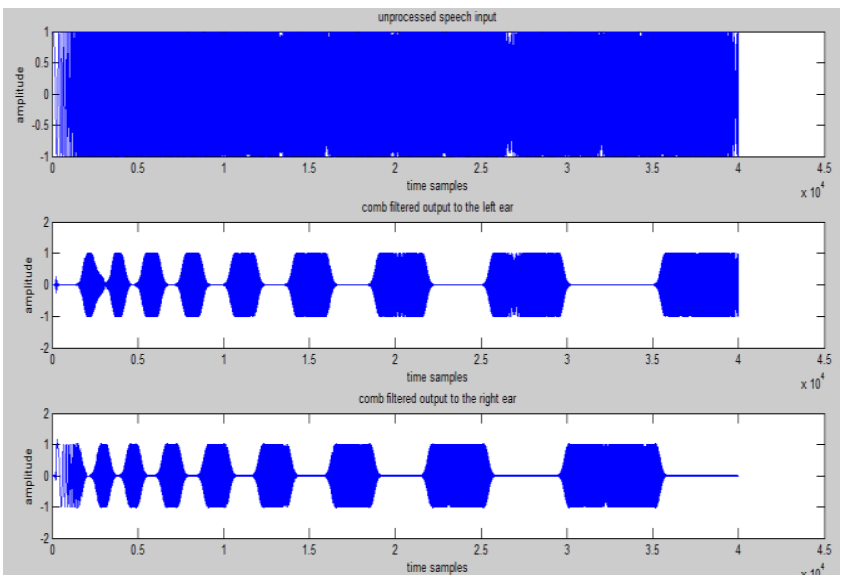

Fig.4. Unprocessed signal, Processed ouput of even bands for right ear and Processed ouput of odd bands for left ear having input 'chirp' signal

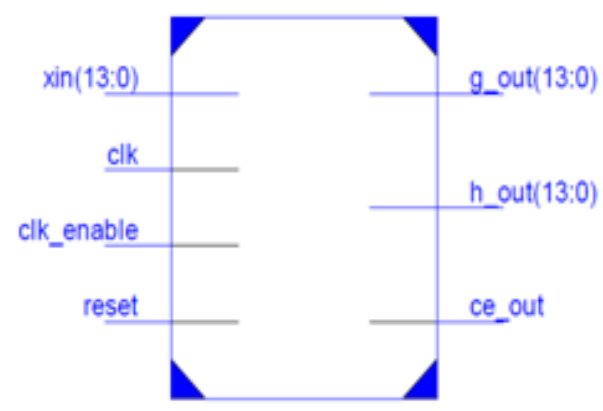

Fig.5. RTL schematic for critical band filter

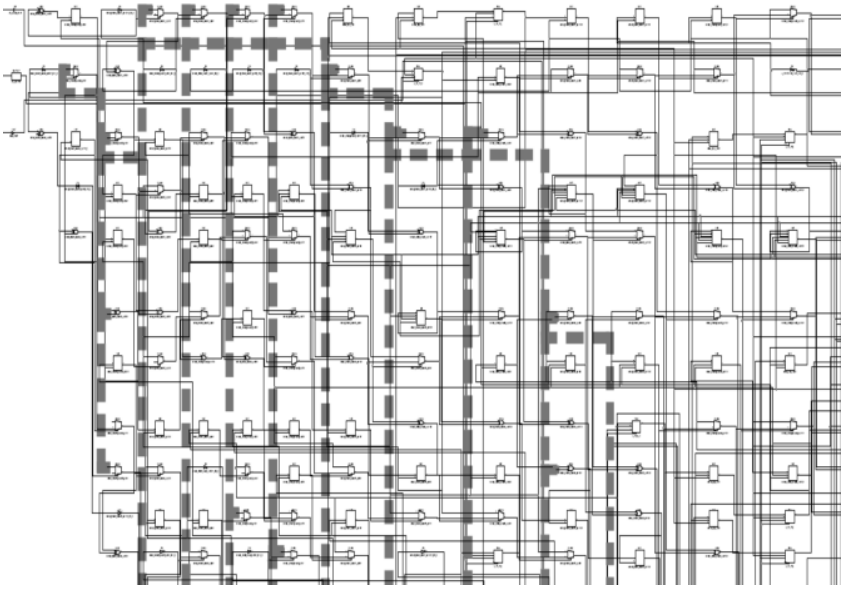

Fig.6. Detailed schematic of RTL

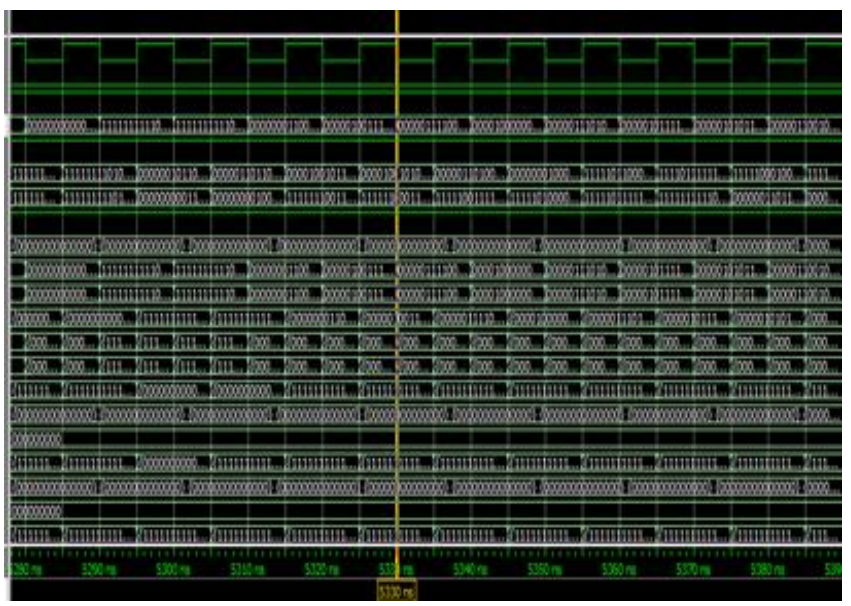

Fig.7. Simulated test bench waveforms of comb filter

The averaged power spectrums for unprocessed and processed test speech signal are observed, as shown in Fig.8. The PSD of the processed signal was obtained in the complementary form with respect to each other. The advantage of using the power spectrum is that high amplitude spectral peaks, which gives more information about the place feature of articulation, are emphasized more than the smaller peaks.

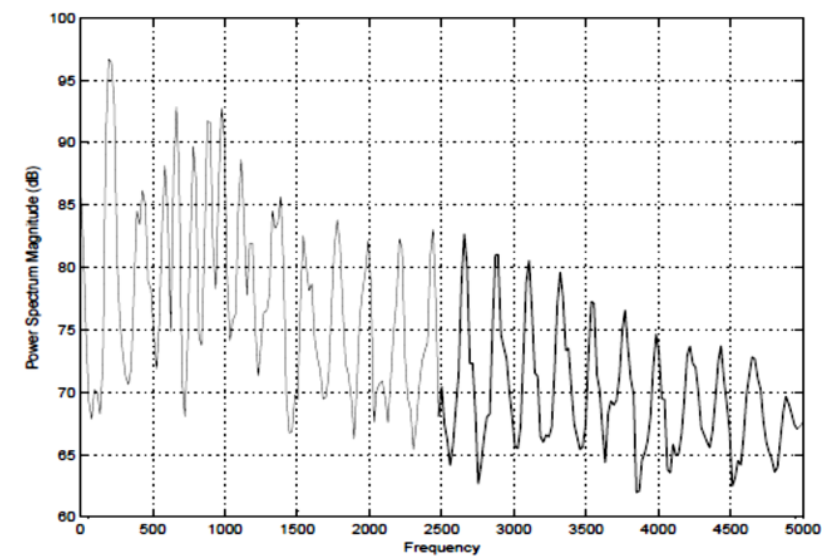

(a) 


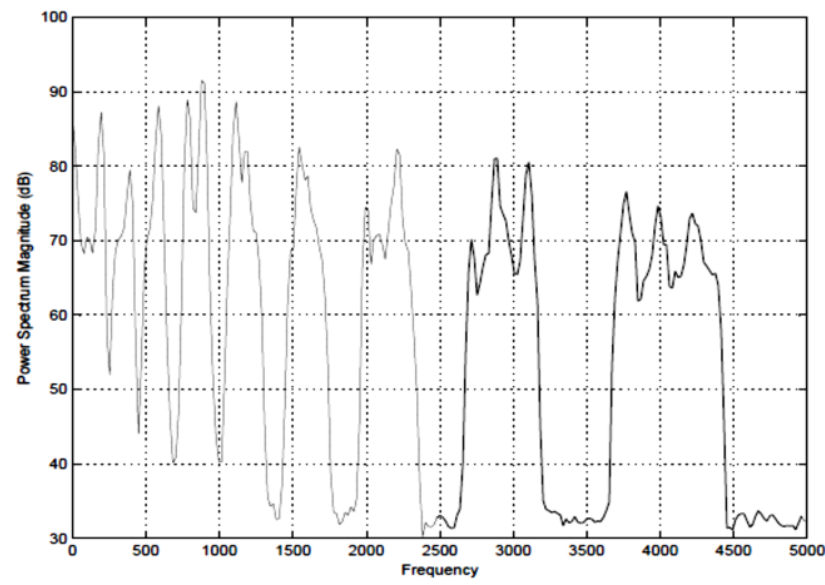

(b)

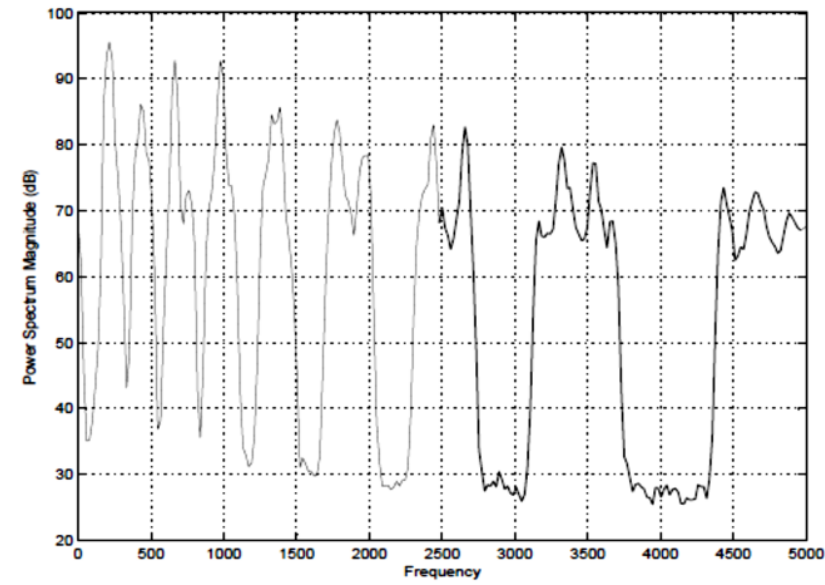

(c)

Fig.8. (a) Power spectral density of unprocessed signal (b) obtained power spectral density of even band for right ear (c) obtained power spectral density from odd band for left ear

The Fig.9 shows the experimental setup for FPGA implementation. For implementation the Xilinx spartan6 XC6SLX45 CSG324 FPGA from Digilent has been used. The FPGA board is connected to the computer system via Ethernet and USB cable. The samples are taken from the computer and being applied to the FPGA board by means of Ethernet cable. The signal is processed in FPGA and the processed output signal is available using Ethernet cable on computer.

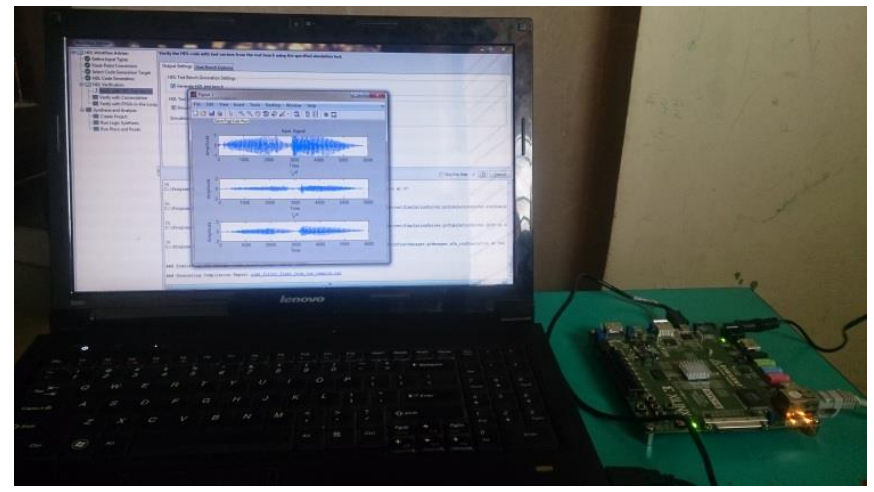

Fig.9. Experimental Setup

\section{CONCLUSION}

A comb filter based on auditory critical bandwidth having 512 coefficients is designed and implemented on FPGA. The developed algorithm uses set of filter coefficients, which are obtained from frequency sampling technique. A new approach helps to implement this comb filter on FPGA, with no conversion complexity making it a significant improvement. Interestingly, the magnitude response of these filters is optimized for low perceived spectral distortion by having low pass band ripple and high stop band attenuation, hence useful for moderate loss sensorineural hearing-impaired people. The low resource requirement of FPGA design shows the significance of the method, which can be effective for development of binaural hearing aids.

\section{REFERENCES}

[1] B. Moore, “An Introduction to Psychology of Hearing", $6^{\text {th }}$ Edition, Brill, 2013.

[2] L. R. Rabiner and R. W. Schafer. "Digital Processing of the Speech Signals", Prentice Hall, 1978.

[3] J.G. Proakis and D.G. Manolakis, "Digital Signal Processing Principles, Algorithms, and Applications", Prentice Hall, 1997.

[4] P.N. Kulkarni and P.C. Pandey. "Optimizing the Comb Filters for Spectral Splitting of Speech to Reduce the Effect of Spectral Masking”, Proceedings of International conference on signal processing, Communication and Networking, pp. 69-73, 2008.

[5] T. Lunner and J. Hellgren, "A Digital Filter Bank Hearing Aid- Design, Implementation, and Evaluation", Proceedings of IEEE International Conference on Acoustics, Speech, and Signal Processing, pp. 3661-3664, 1991.

[6] D.S. Chaudhari and P.C. Pandey, "Dichotic Presentation of Speech Signal using Critical Filter Bank for Bilateral Sensorineural Hearing Impaired", Proceedings of $16^{\text {th }}$ International Conference on Acoustics, pp. 213-214, 1998.

[7] E.W. Zwicker, "Subdivision of Audible Frequency Range into Critical Bands (Freqenzgruppen)", The Journal of the Acoustical Society of America, Vol. 33, pp. 248-253, 1961.

[8] Lendra M. Friesen, Robert V. Shannon, Deniz Baskent and Xiaosong Wang, "Speech Recognition in Noise as a Function of the Number of Spectral Channels: Comparison of Acoustic Hearing and Cochlear Implants", The Journal of the Acoustical Society of America, Vol. 110, No. 2, pp. 11501156, 2001.

[9] J.R. Dubno and A.B. Schaefer, "Comparison of Frequency Selectivity and Consonant Recognition among HearingImpaired and Masked Normal-Hearing Listeners", The Journal of the Acoustical Society of America, Vol. 91, No. 4, pp. 2110-2121, 1992.

[10] Nivin Ghamry, "FPGA Implementation of Hearing Aids using Stationary Wavelet-Packets for Denoising", International Journal of Computer Applications, Vol. 69, No. 15, pp. 30-36, 2013

[11] Matlab Documentation, Available at: https://in.mathworks.com/help/?requestedDomain=www.m athworks. 
[12] Salaheddine Derouiche and Bachir Djedou, "Implementation of the Development of Filtering Algorithm to Improve the System of Hearing in Hearing Impaired with Cochlear Implant", Proceedings of Advanced Information Technologies and Applications, pp. 65-79, 2013.
[13] S.G. Kambalimath, P.C. Pandey, P.N. Kulkarni, S.S. Mahant-Shetti and S.G. Hiremath, "FPGA-based Implementation of Comb Filters using Sequential MultiplyAccumulate Operations for use in Binaural Hearing Aids Spectral Masking", Proceedings of IEEE Annual India Conference, pp. 1-5, 2014. 